\title{
Manufacturing of thin glass shells for future space telescopes
}

\author{
Gabriele Vecchi \\ S. Basso, M. Civitani, M. Ghigo, G. Pareschi, B. Salmaso \\ INAF-Osservatorio Astronomico di Brera, ITALY
}




\section{Outline}

Motivation and overview of the manufacturing method

1. Hot Slumping technology of thin glass foils

2. Sub-aperture Polishing/Figuring applied to slumped glass shells

Figuring test on a $130 \mathrm{~mm}$ slumped glass prototype

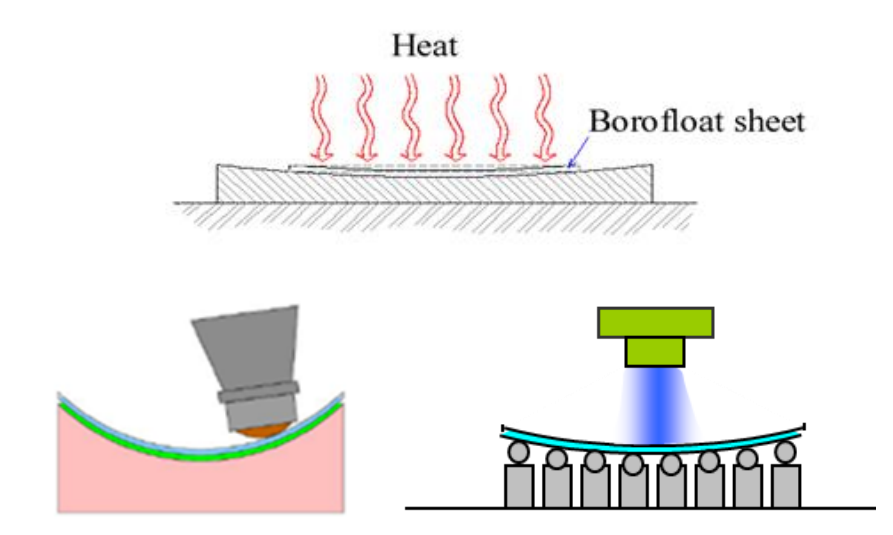

$>$ Metrology

$>$ Temporary stiffening

$>$ Surface accuracy and micro-roughness results

Conclusions

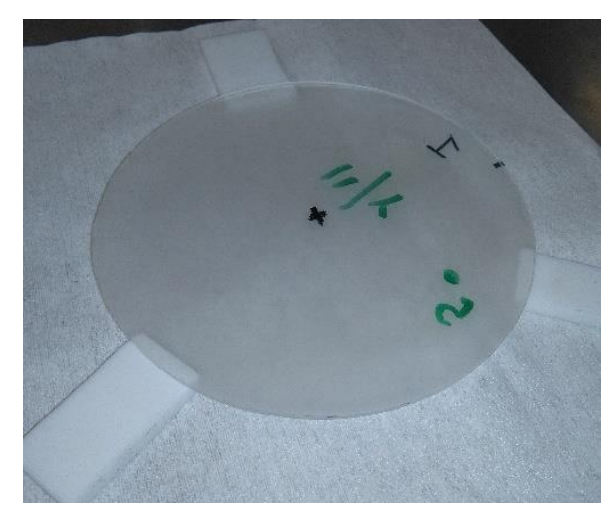




\section{Motivation}

$\checkmark$ Lightweight optics are needed for space applications

- Thin shells of glass used in adaptive optics are relevant for active optics in space

GRINDING/POLISHING

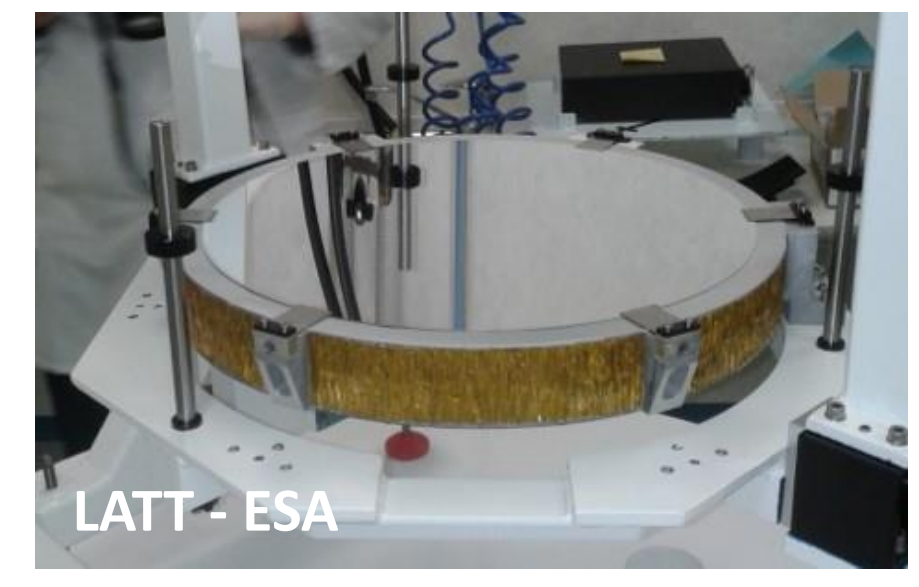

Start the process from thin foils and skip the laborious step of thinning of polished optics

- Thin foils of glass are commercially available High-quality, thin $(1 \mathrm{~mm})$, large $(1 \mathrm{~m})$

Shaping foils by slumping technology Replicated optics for large segmented mirrors

$\checkmark$ Know how built at INAF-OAB making glass shell prototypes for adaptive optics by Hot Slumping technology

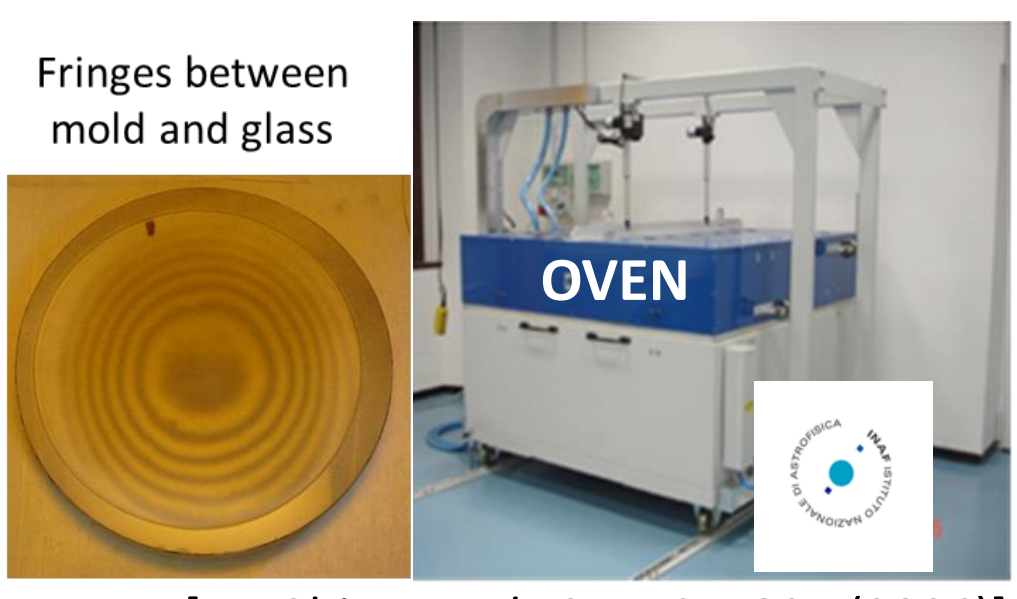

[M. Ghigo et al., SPIE 61480E (2006)] 


\section{Hot Slumping technology}

$\checkmark$ The glass sheet is placed into the oven, above a mandrel (mould) of complementary target shape.

$\checkmark$ With a suitable thermal cycle the glass softens enough to slump onto the mandrel surface and copy its shape.

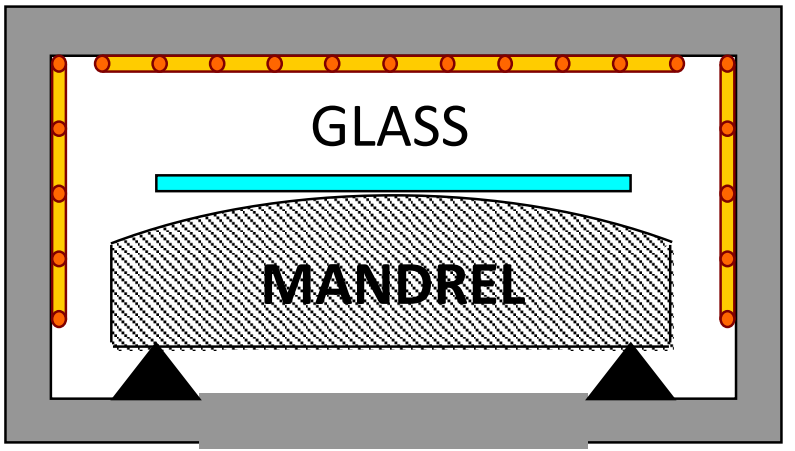

$$
\text { slump onto the mandrel surface and copy its shape. }
$$

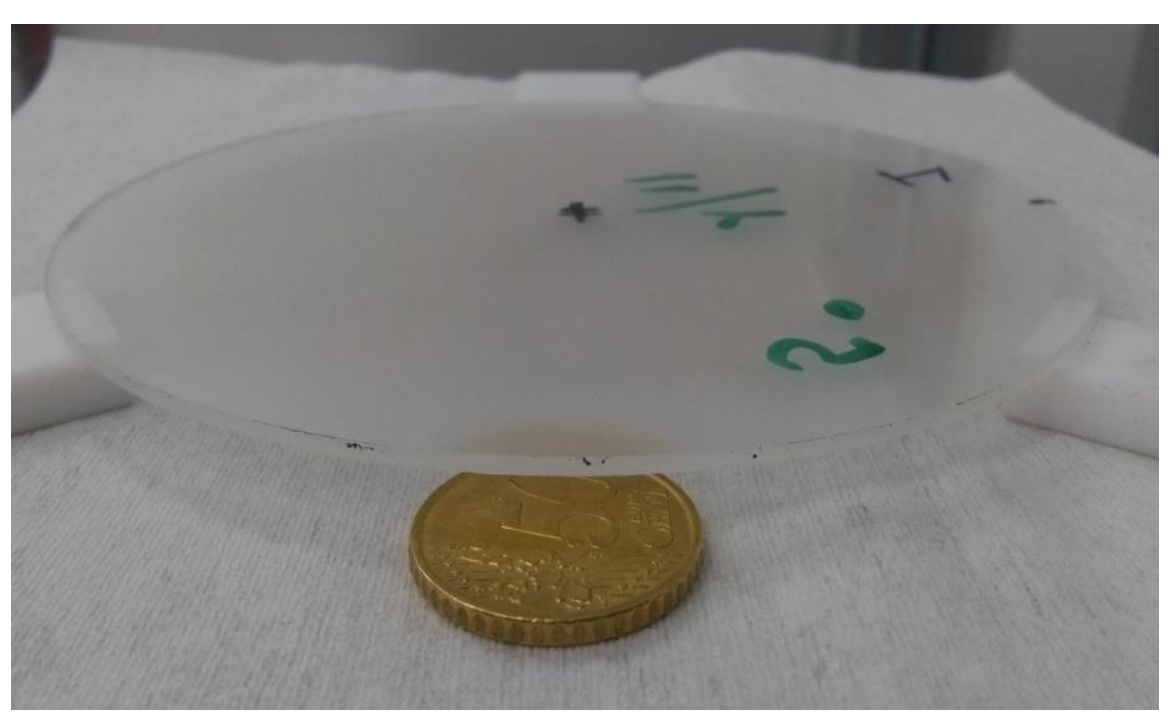

- BOROFLOAT ${ }^{\circledR} 33$ borosilicate glass (Schott).

- Circular disks of $130 \mathrm{~mm}$ diameter, thickness of $2 \mathrm{~mm}$, 1:65 thickness-to-diameter ratio.

$\square 4 \mathrm{~m}$ Radius of Curvature spherical shells. 


\section{Thin glass slumped shell: Metrology}

1. FEM assessment of support-induced deformations.

2. Shell supported on three points.

3. Zygo GPI Interferometer to measure the surface form error.
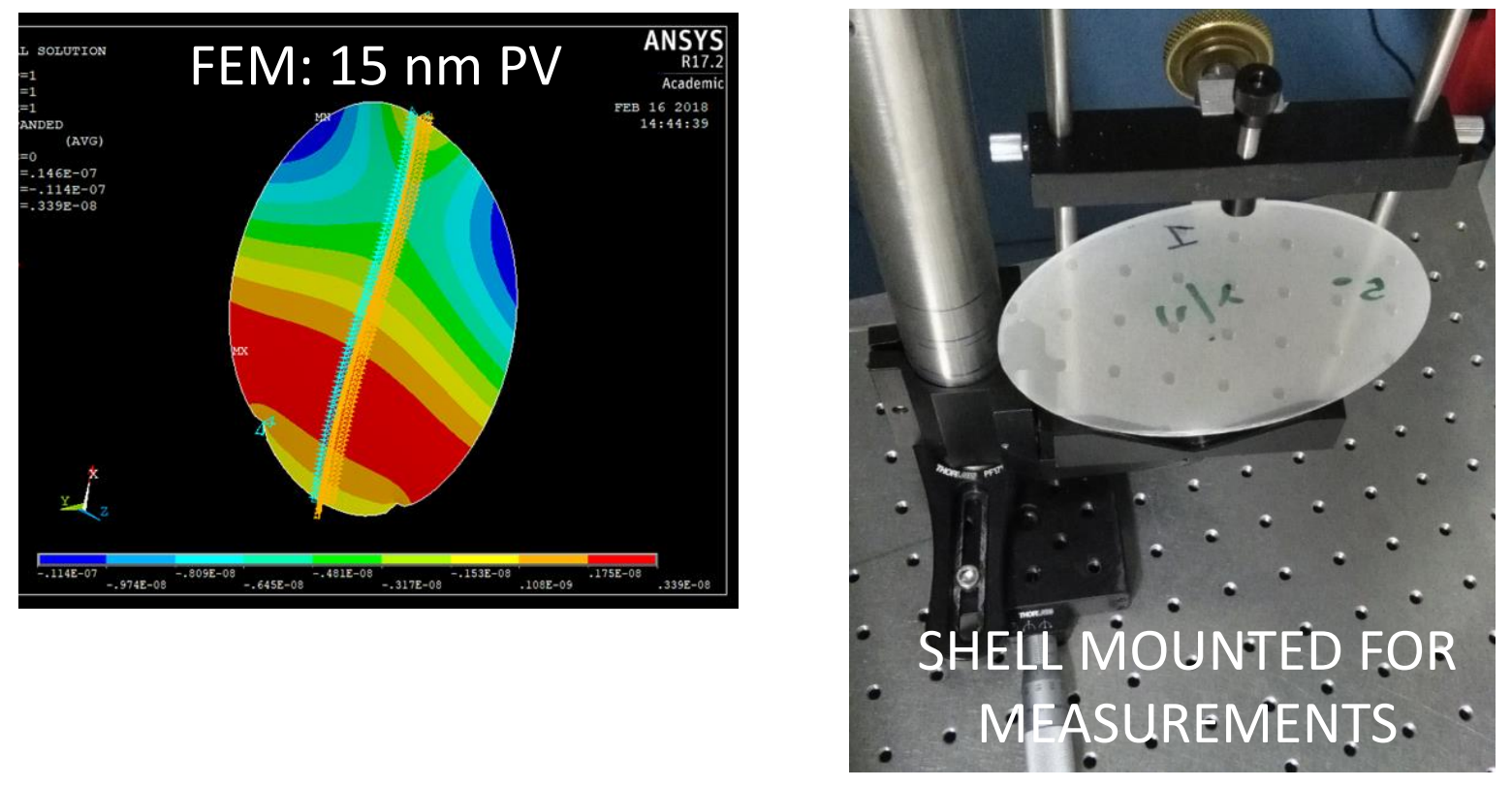

Measured error map

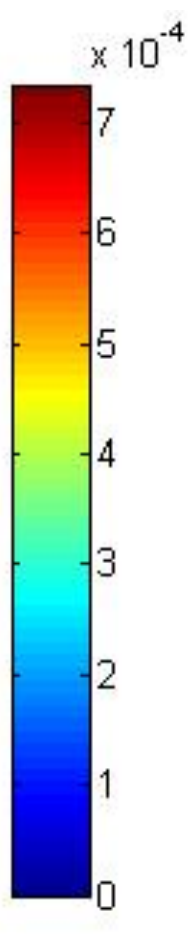

Repeatability: $12 \mathrm{~nm} \mathrm{RMS}^{5}$

$128 \mathrm{~nm}$ RMS, $736 \mathrm{~nm} \mathrm{PV}$

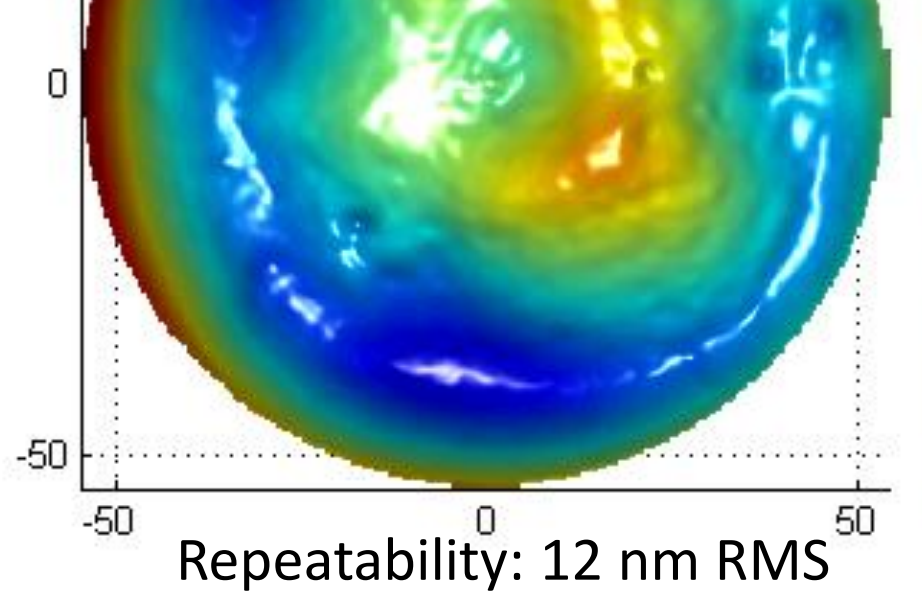

Shape accuracy (w/o tilt, power) reaches $\sim \lambda / 5$ RMS (> $\lambda$ PV ) over $110 \mathrm{~mm}$.

Further improvement of the surface accuracy is required. 


\section{Trade off}

\section{Slumping optimization}

- Improving the figure accuracy of the mould and optimizing the process parameters would return better slumped shells

- Thermal gradients in the glass are sources of residual shape errors, and they are difficult to eliminate completely

\section{Post-slumping Figuring: Bonnet and lon}

Figuring + metrology on flexible glass shells
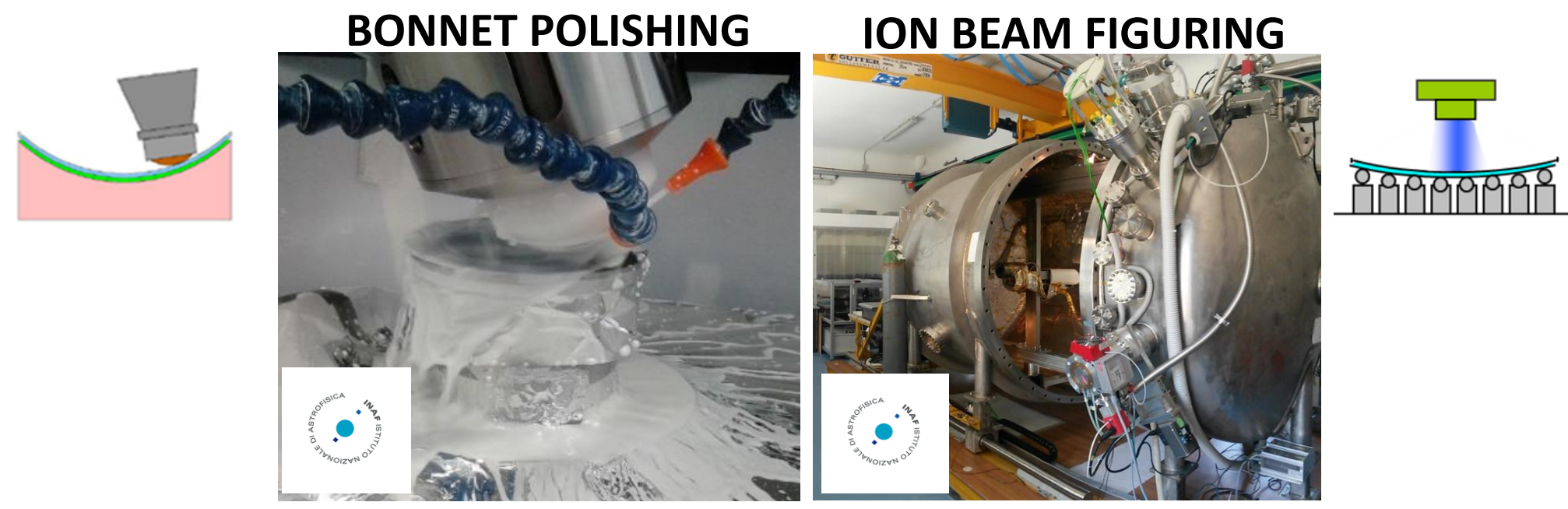

- Mitigation of requirements by figure-control (low freq.) in active optics system

- Contactless lon figuring to avoid print-through (lightweighting) and correct edges (segmentation) 


\section{Bonnet Polishing/Figuring Technology}

GOAL: to improve the surface accuracy of thin slumped shells

IRP1200 by Zeeko Ltd. is a 7 axis CNC machine
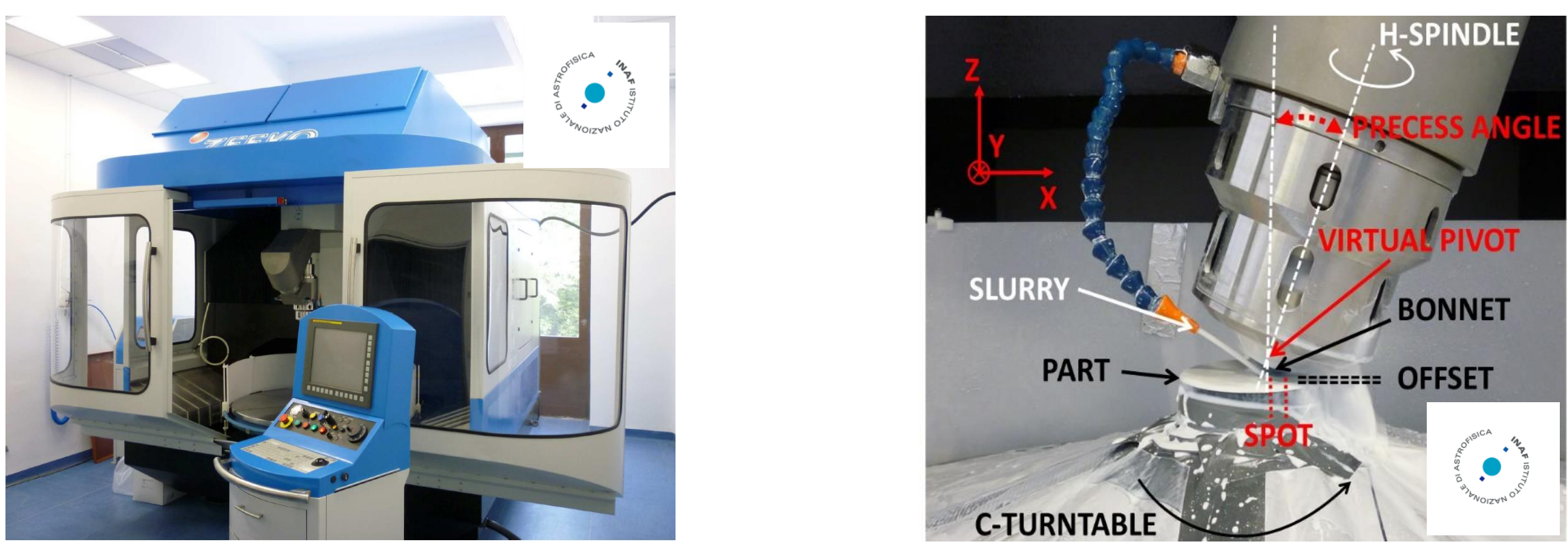

* The bonnet is an inflated spinning tool in contact with the optical surface

* Removal is proportional to dwell time, relative surface speed and pressure (Preston model)

* The dwell time map is derived from surface metrology 


\section{Temporary stiffening of the thin shell}

During bonnet polishing the shell is fixed to a holder
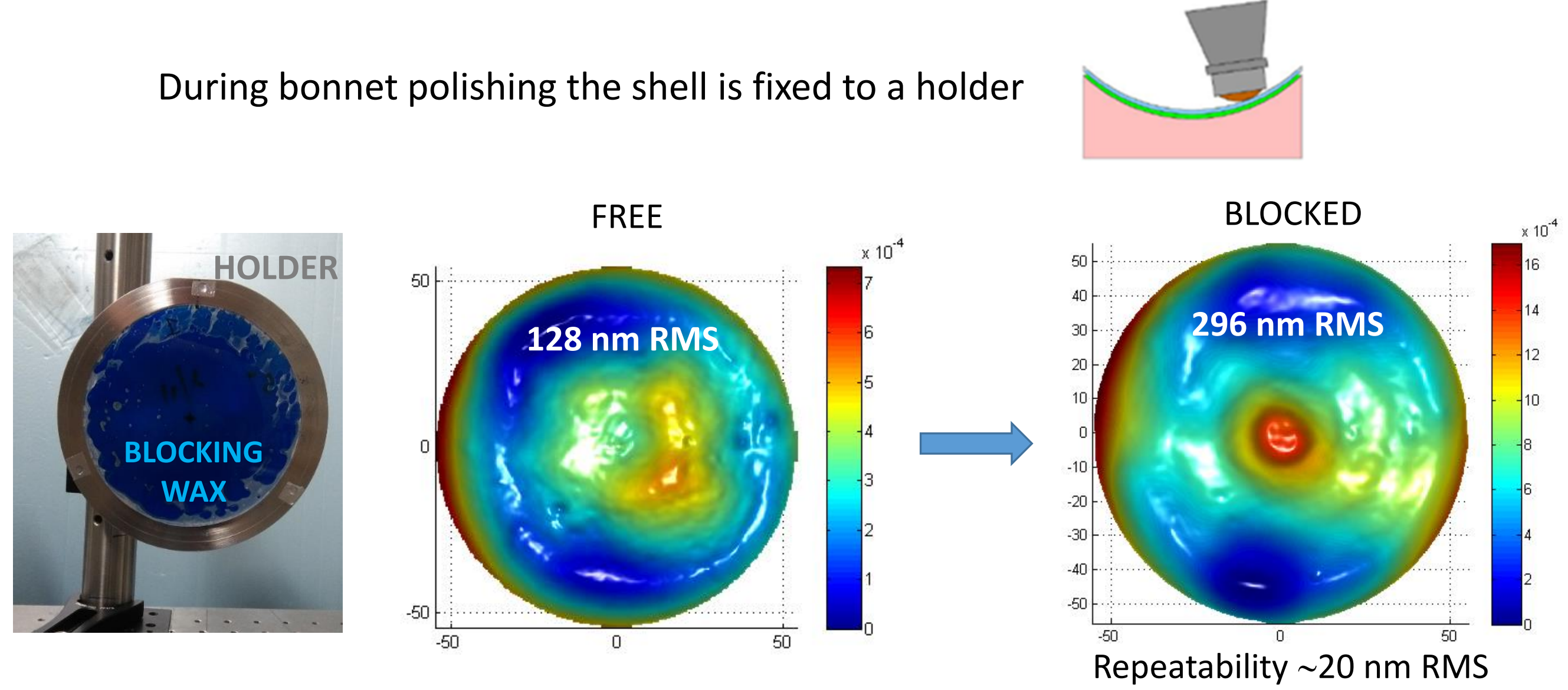

Non-optimized matching between holder and glass shell

Test the metrology on the blocked shell guiding the figuring process

> Assess any print-through related to the temporary deformation of the shell 


\section{Figuring of the slumped shell - I}

Trend of expected error map from differential measurements on the blocked shell.
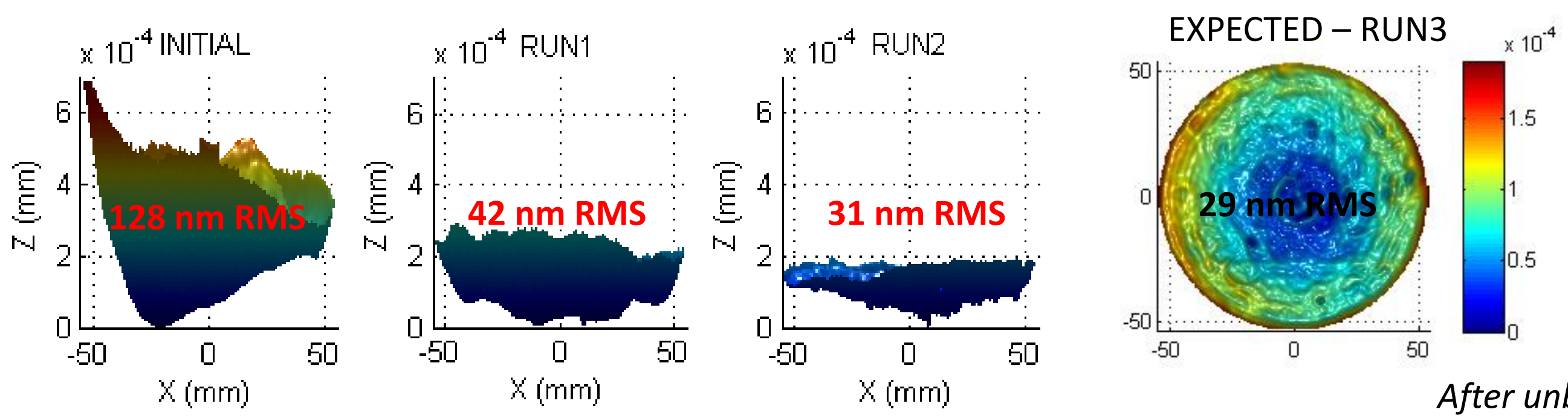

- Overall figuring time: $\sim 2 \mathrm{~h}$, wavelength error $>10-20 \mathrm{~mm}$ addressed

- Glass shell released from holder at the end of the corrective process for final metrology

- Shape accuracy (w/o tilt, power) improved from $\sim \lambda / 5$ RMS to $\sim \lambda / 20$ RMS over $110 \mathrm{~mm}$.

- Metrology accuracy on the blocked shell limited by large deformation

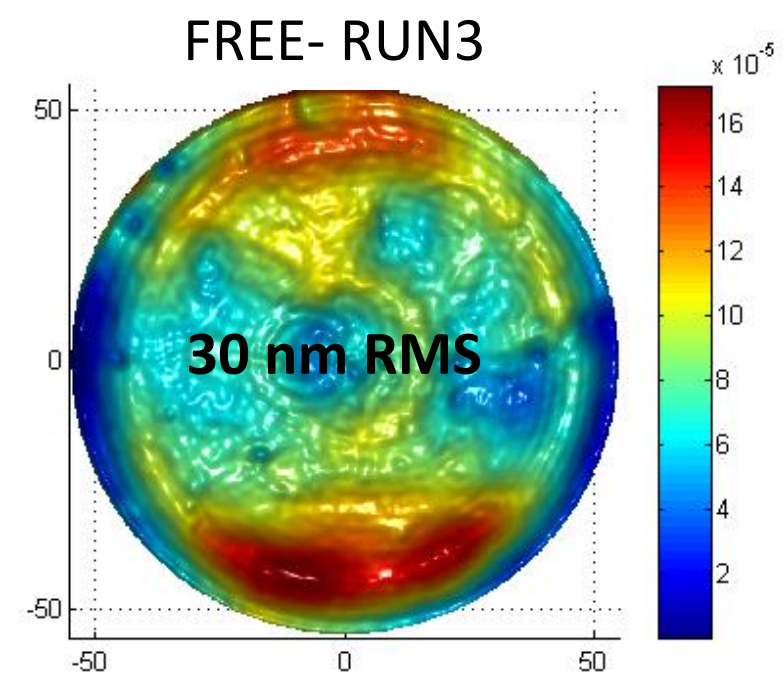

$10 \mathrm{~nm}$ RMS repeatability 


\section{Deformation of the thin shell}

Improve the mechanical matching between holder and glass shell

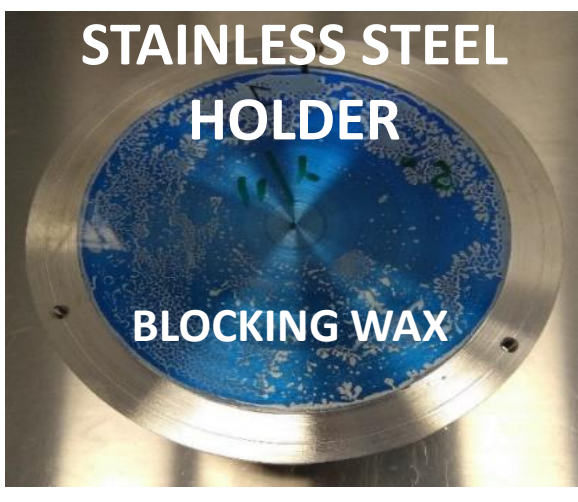

Holder was reworked to fit better to the shell shape
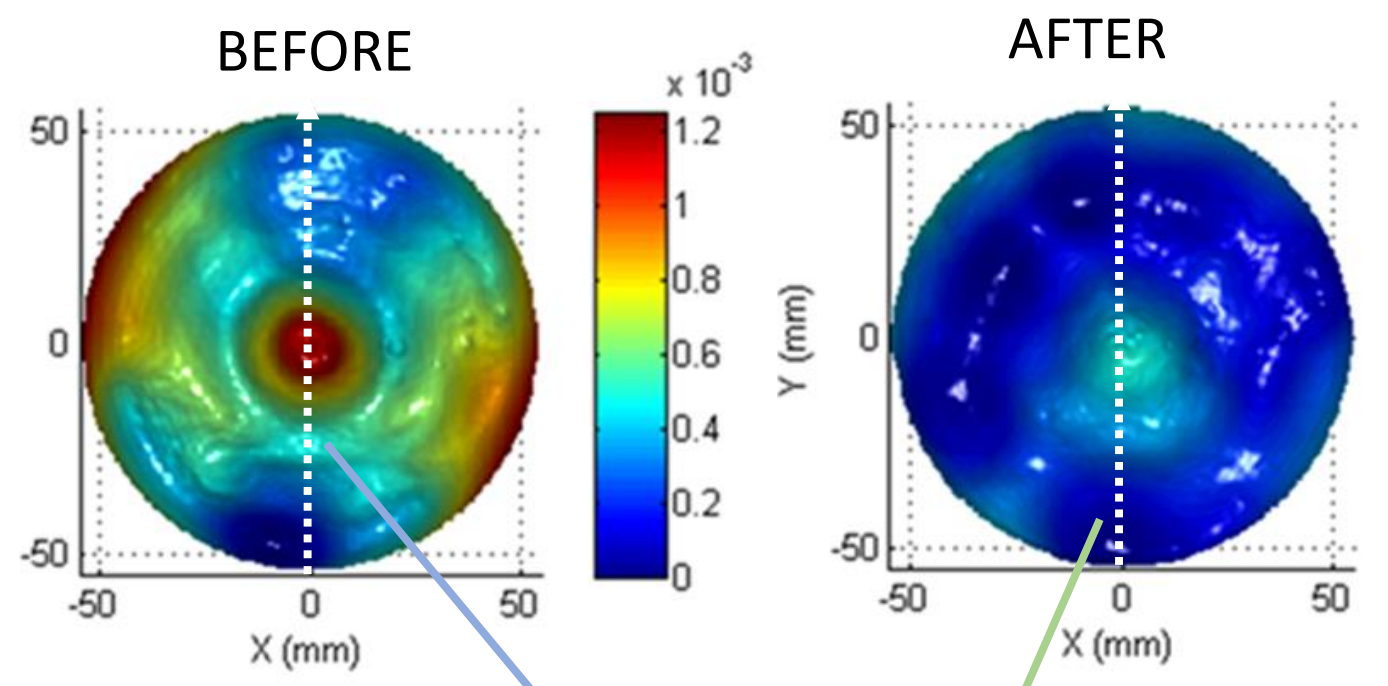

Error maps measured on the blocked shell

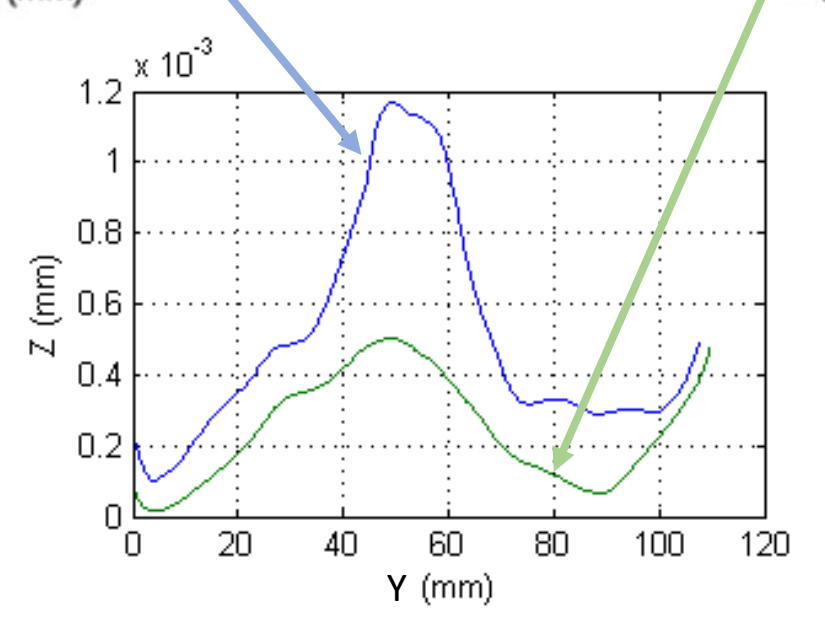

After reworking the stiffening holder, the error map of the blocked shell decreased by more than a factor two

- from $221 \mathrm{~nm}$ to $106 \mathrm{~nm}$ RMS

- from $\sim 1200 \mathrm{~nm}$ to $\sim 500 \mathrm{~nm}$ PV

Further improvement is possible

Reduce the risk of print-through effect

$>$ Improve the metrology accuracy throughout the figuring process 


\section{Figuring of the slumped shell - II}

Error map measured on the free standing shell.

RUN5 - Profiles of the residual error map

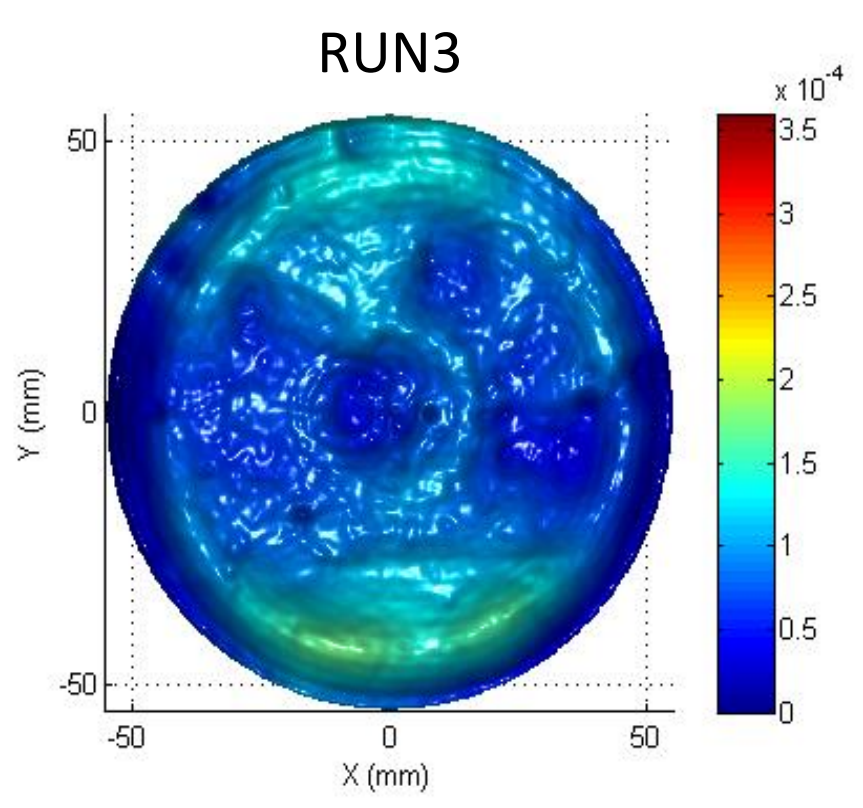

30 nm RMS \& 170 nm PV

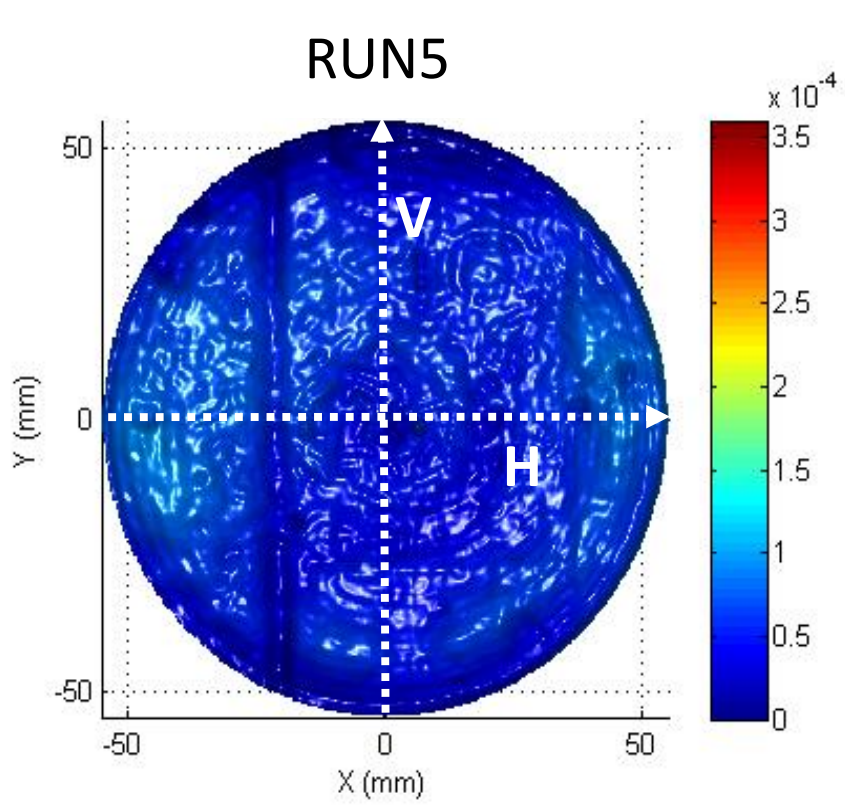

15nm RMS \& 105 nm PV

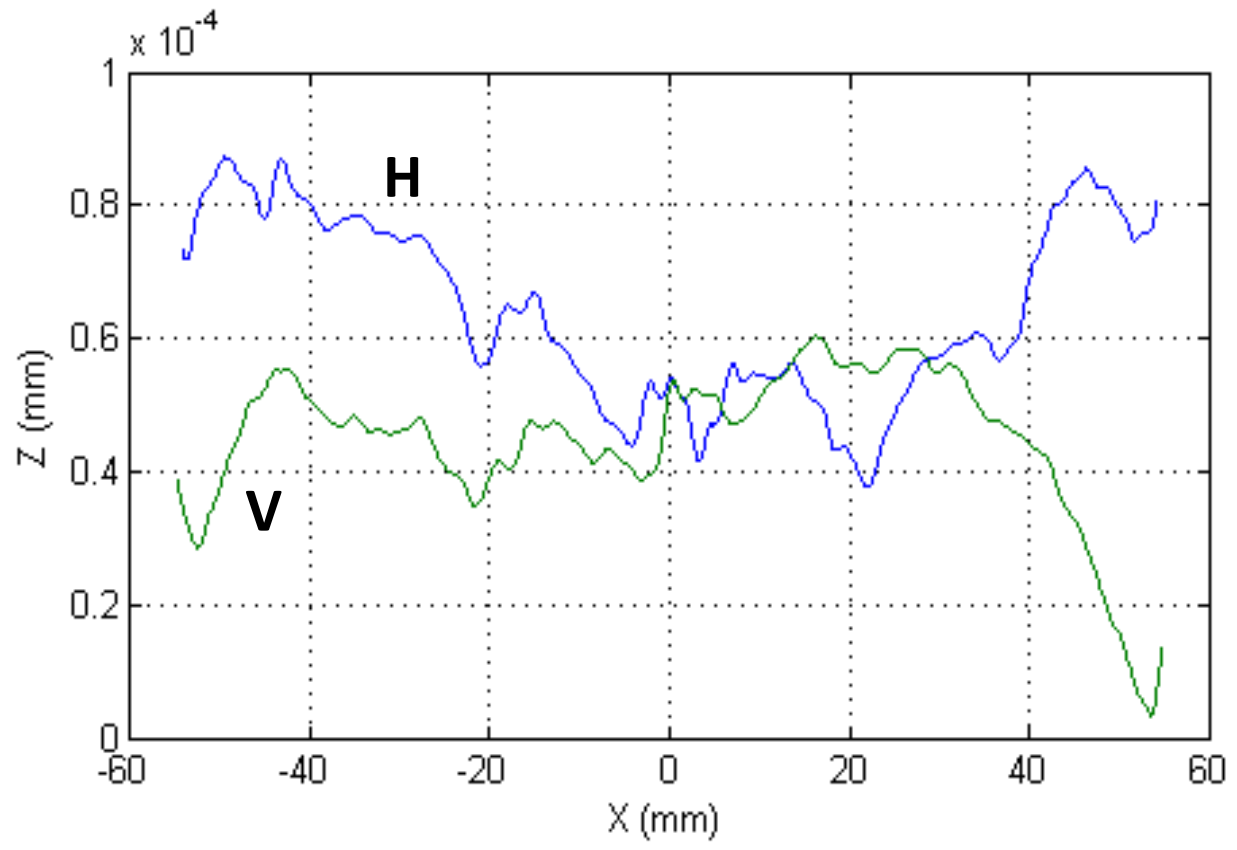

$>$ Overall figuring time: $\sim 4 \mathrm{~h}$, wavelength error $>10 \mathrm{~mm}$ addressed

$>$ Shape accuracy (w/o tilt, power) improved from $\sim \lambda / 20$ RMS (RUN3) to $\sim \lambda / 42$ RMS and $\sim \lambda / 6$ PV (RUN5) over $110 \mathrm{~mm}$

Smoothing of mid spatial frequencies $(<5 \mathrm{~mm})$ is effective using, e.g., pitch-based process 


\section{Figuring of the slumped shell - II}

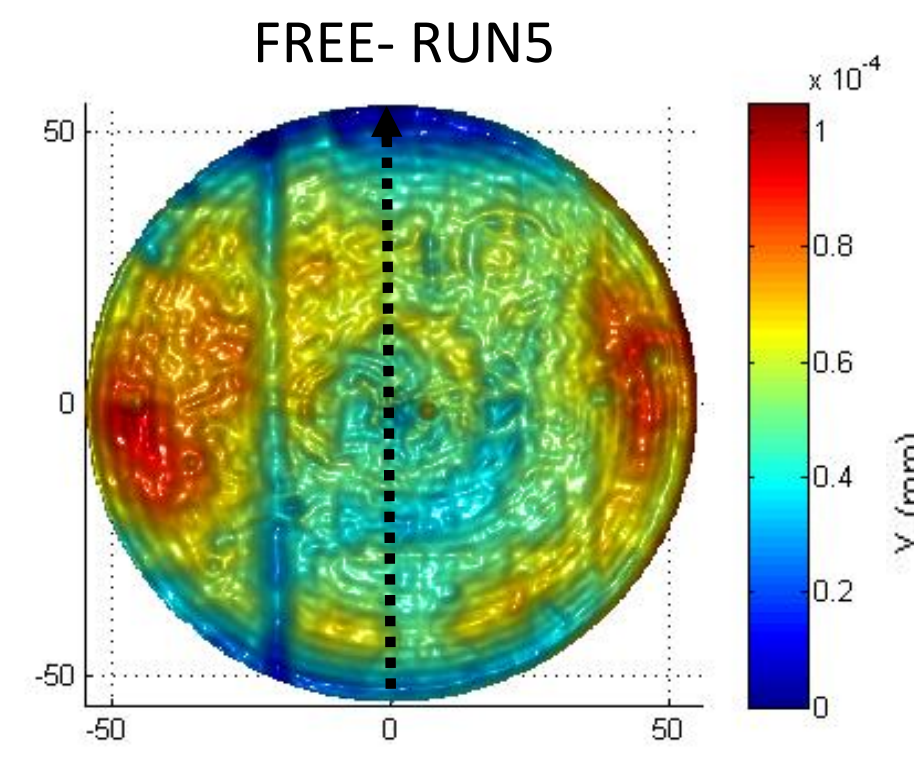

15nm RMS \& $105 \mathrm{~nm}$ PV

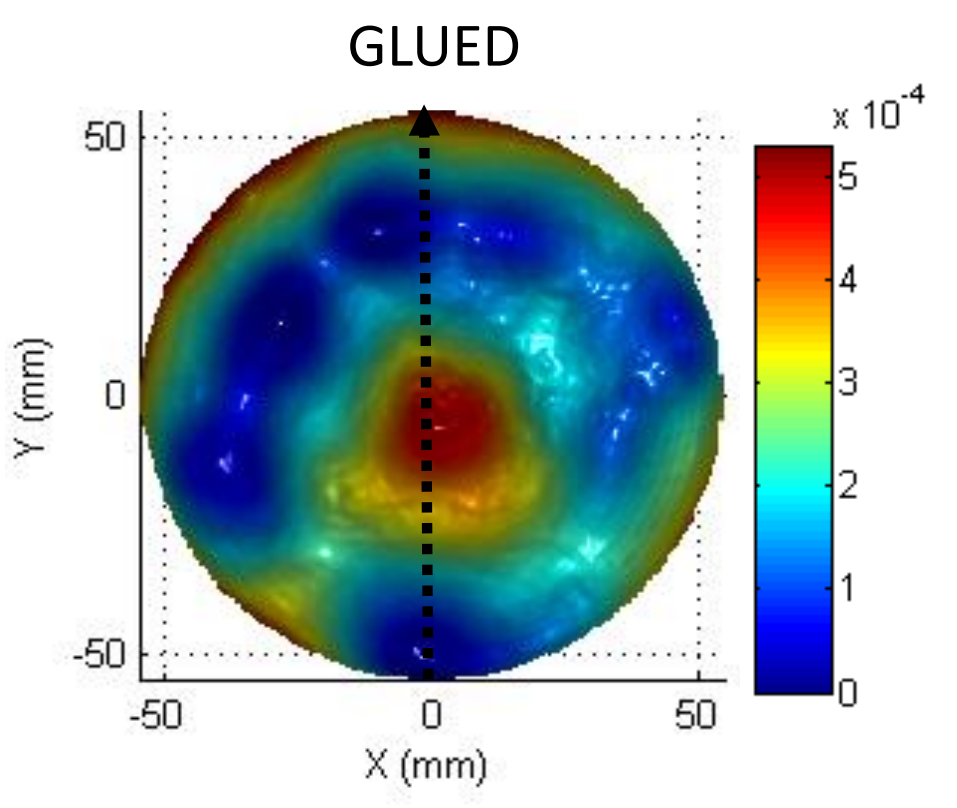

106 nm RMS \& 500 nm PV
Error map profiles measured on the shell in blocked and free standing configuration

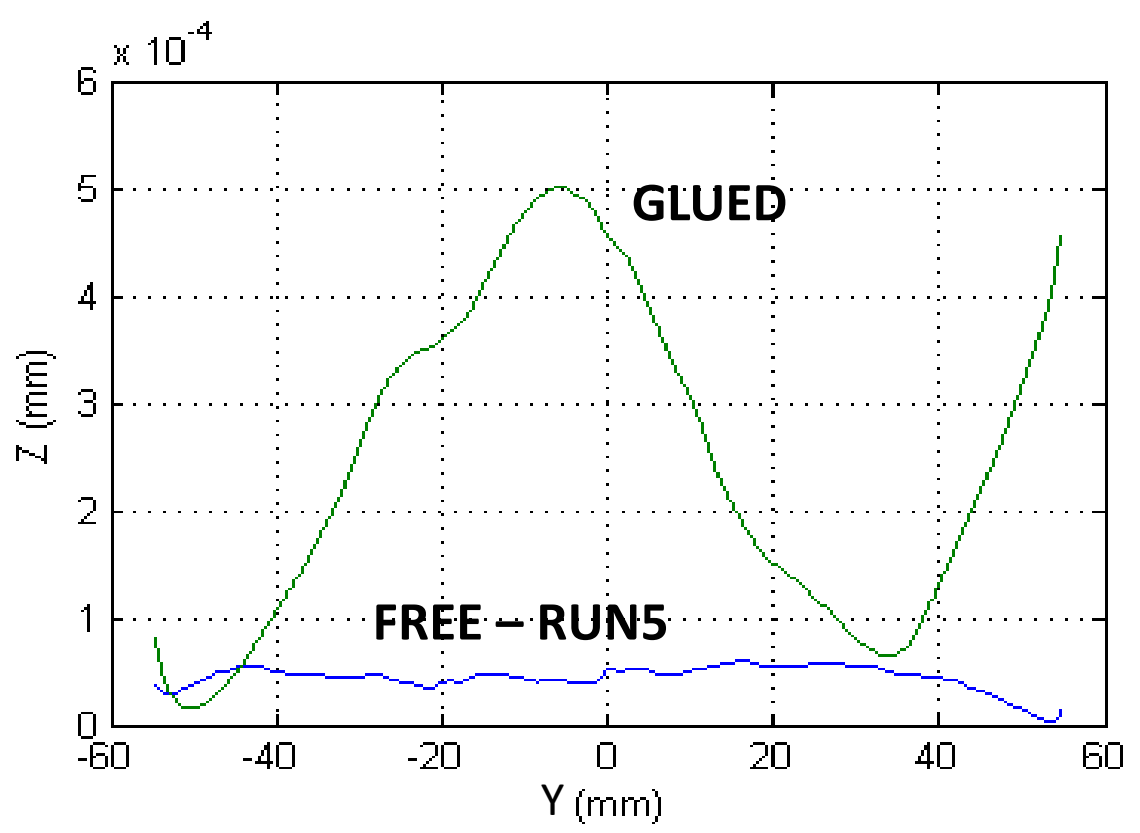

$\checkmark$ No significant print-through occurred in run 4-5 due to the temporary deformation of the shell

$\checkmark$ The vertical groove was probably induced by a perturbation occurred to the polishing process

$\checkmark 15 \mathrm{~nm}$ RMS achieved is close to the figuring requirement before active compensation 


\section{Micro-roughness}

Measured with Micro-Finish Topographer, 10X objective, FOV: 0,72 ×0,96 mm²

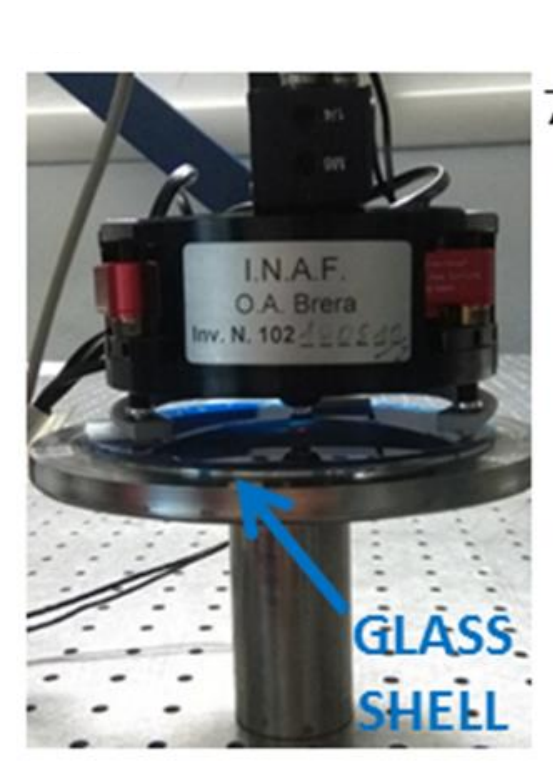

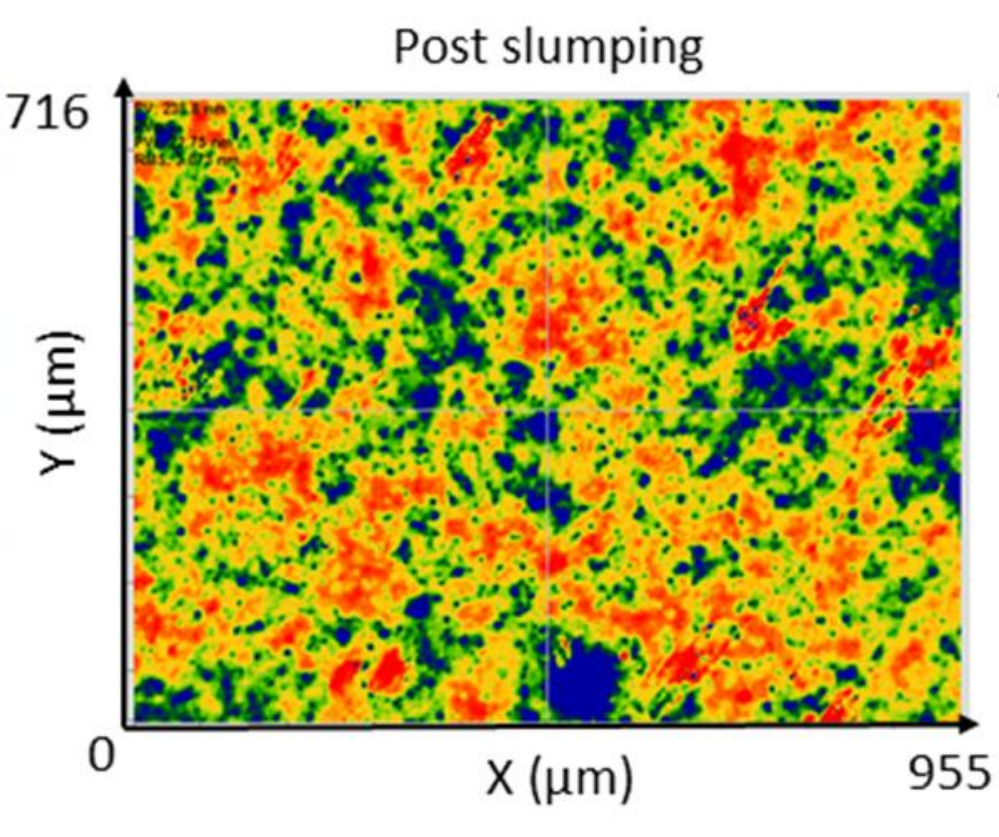

3 nm RMS

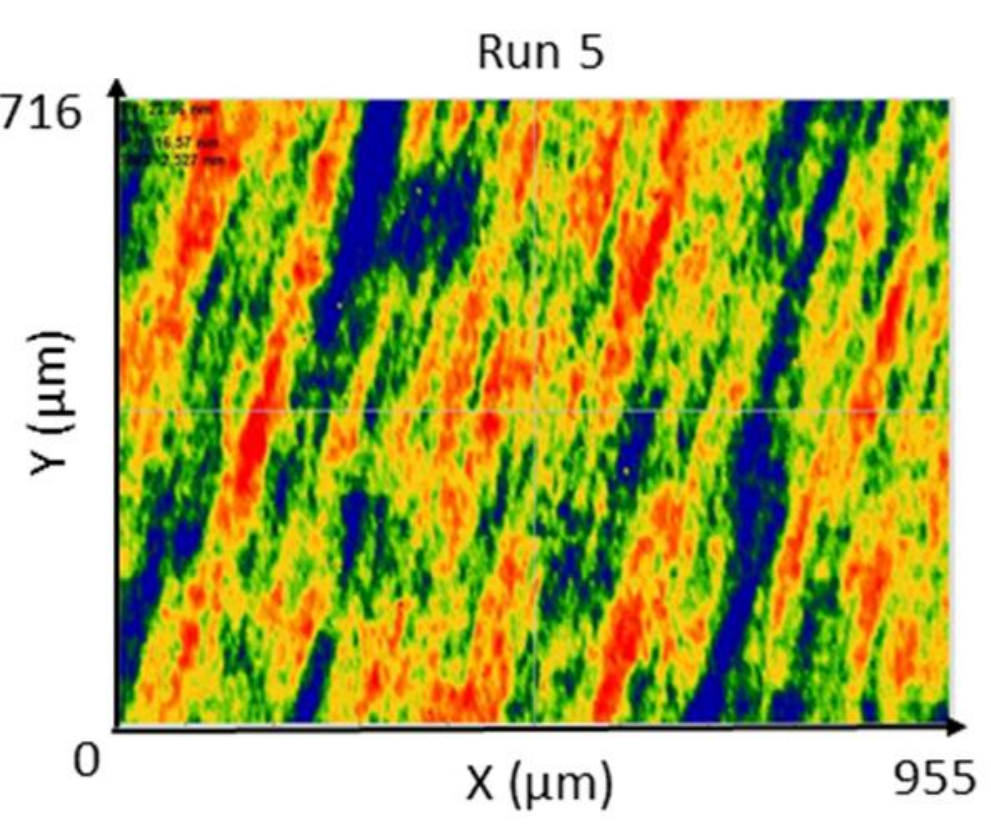

$\sim 2.5 \mathrm{~nm}$ RMS

We monitored the micro-roughness trend, although the process was not optimized to reduce it.

Micro-roughness is expected to improve by switching to finer abrasive. 


\section{Conclusions}

- At INAF-OAB we are combining hot slumping and polishing/figuring technologies to develop a manufacturing method of thin shells of glass, relevant for active optics in space.

- The method benefits of

High quality thin glass foils available on the market

* Replication of the mould shape

> Metrology capability drives the manufacturing process. Repeatability of $10 \mathrm{~nm}$ RMS is achieved with a simple setup measuring $130 \mathrm{~mm}$ shells.

$>$ Bonnet polishing improved significantly the shape accuracy on $130 \mathrm{~mm}$ slumped glass shell, approaching the figuring requirement.

$\sim \lambda / 5$ RMS $\rightarrow \sim \lambda / 42$ RMS over $110 \mathrm{~mm}$ 